\title{
Perfil nutricional de usuários do Programa HIPERDIA em Ananindeua, Pará, Brasil
}

\author{
Nutritional profile of the HIPERDIA Program users in Ananindeua, Pará state, Brazil \\ Perfil nutricional de los usuarios del Programa HIPERDIA en Ananindeua, Pará, Brasil
}

\author{
Bruna Ranyelle de Marinho Sousa. Universidade Federal do Pará (UFPA). Belém, PA, Brasil. bruna-ranyelle@hotmail.com (Autora correspondente) \\ Domitila Pereira Blanco Vieira. Universidade Federal do Pará (UFPA). Belém, PA, Brasil. domi-blanco@hotmail.com \\ Isameriliam Rosaúlem Pereira Silva. Universidade Federal do Pará (UFPA). Belém, PA, Brasil. rosaulen@hotmail.com \\ Tainá Pinheiro Braga. Universidade Federal do Pará (UFPA). Belém, PA, Brasil. tata_pb@hotmail.com \\ Giselle Cristina dos Santos Burçãos. Prefeitura Municipal de Ananindeua. Ananindeua, PA, Brasil. giselleburcaos@gmail.com \\ Cláudia Daniele Tavares Dutra. Universidade Federal do Pará (UFPA). Belém, PA, Brasil. cdani@ufpa.br \\ Carla Andréa Avelar Pires. Universidade Federal do Pará (UFPA). Belém, PA, Brasil. carlaavelarpires@bol.com.br
}

\section{Resumo}

Objetivo: Investigar o perfil nutricional, conhecer os hábitos alimentares de pacientes do programa HIPERDIA e contribuir para a discussão sobre medidas preventivas e a promoção da saúde na atenção primária. Métodos: Durante o período de agosto de 2011 a fevereiro de 2012, alunos e preceptores do Programa de Educação pelo Trabalho para a Saúde (PET-Saúde) da Universidade Federal do Pará entrevistaram 125 indivíduos hipertensos e/ou diabéticos, cadastrados na Estratégia Saúde da Família do "Jardim Florestal" e "Mururé", no município de Ananindeua, Pará. Foi utilizado questionário estruturado previamente elaborado, contendo perguntas sobre o perfil socioeconômico, dados clínicos e hábitos/estilos de vida referentes à alimentação. Resultados: Entre os entrevistados, $65,6 \%$ eram diabéticos e $84,8 \%$ hipertensos. A maioria $(81,6 \%)$ dos entrevistados informou estar fazendo alguma dieta ou cuidado com a alimentação para auxiliar no controle da hipertensão arterial ou do diabetes mellitus, havendo, de acordo com eles, a redução principalmente de sal, gordura e açúcar. 0 sobrepeso (IMC acima de 24,9kg/m²) foi um fator predominante na população, atingindo 73,6\% dos entrevistados. Conclusão: Verificou-se que os entrevistados não estavam desempenhando um cuidado adequado com a alimentação. Nesse contexto, torna-se necessário melhorar o acompanhamento nutricional, bem como implementar medidas preventivas socioeducativas para controlar as doenças crônicas não transmissíveis, beneficiando assim a qualidade de vida das pessoas.

\section{Abstract}

Objective: To investigate the nutritional profile and understand the dietary habits of patients in the HIPERDIA Program aiming to contribute to the discussion on disease prevention and health promotion in primary care. Methods: From August 2011 to February 2012, students and tutors of the "Programa de Educação pelo Trabalho para a Saúde" (PET-Saúde) of the Federal University of Pará interviewed 125 individuals with hypertension and/ or diabetes registered in the Family Health Strategy of "Jardim Florestal" and "Mururé" in the municipality of Ananindeua, Pará state. We applied a structured questionnaire containing questions about socio-economic profile, clinical data, habits, and life style related to patients' food consumption. Results: Among the respondents, $65.6 \%$ were diabetics and $84.8 \%$ were hypertensive. Most of the respondents $(81.6 \%)$ claimed that they had been following a diet to control hypertension or diabetes mellitus, especially with regard to salt, fat, and sugar reduction. Overweight (BMl greater than $24.9 \mathrm{~kg} / \mathrm{m}^{2}$ ) was a predominant factor in the population analyzed (73.6\% of the respondents). Conclusion: The research participants were not following an adequate diet requirement. In this context, it is necessary to improve nutritional monitoring and implement socio-educational preventive measures in order to control chronic non-transmissible diseases, thus ameliorating people's quality of life.

\section{Resumen}

Objetivo: Investigar el perfil nutricional y conocer los hábitos alimentarios de los pacientes del Programa HIPERDIA y contribuir a la discusión sobre la prevención y promoción de salud en la atención primaria. Métodos: Durante el período de agosto 2011 a febrero 2012, los estudiantes y tutores del Programa de Educação pelo Trabalho para a Saúde (PET-Saúde) de la Universidad Federal del Pará, entrevistaron a 125 individuos con hipertensión y/o diabéticos, registrados en la Estrategia de Salud Familiar del "Jardim Florestal" y "Mururé" en la ciudad de Ananindeua, Pará. Se utilizó un cuestionario estructurado previamente diseñado que contenía preguntas sobre la situación socioeconómica, datos clínicos, hábitos y estilos de vida relacionados con la alimentación. Resultados: Entre los encuestados, $65,6 \%$ eran diabéticos y 84,8\% eran hipertensos. La mayoría (81,6\%) de los encuestados afirmaron que estaban haciendo alguna dieta para controlar la hipertensión o la diabetes mellitus, en especial la reducción de sal, grasa y azúcar. El sobrepeso (IMC superior a 24,9 kg/m²) fue un factor predominante: 73,6\% de la población entrevistada. Conclusión: Los encuestados no cuidaban de su alimentación. En este contexto, es necesario mejorar el asesoramiento nutricional, así como implementar medidas preventivas socio educacionales para el control de las enfermedades crónicas no transmisibles, en beneficio de la calidad de vida de las personas.

Como citar: Souza BRM, Vieira DPB, Silva IRP, Braga TP, Burçãos GCS, Dutra CDT et al. Perfil

nutricional de usuários do Programa HIPERDIA em Ananindeua, Pará, Brasil. Rev Bras Med Fam

Comunidade, 2013; 8(28):187-95. Disponível em: http://dx.doi.org/10.5712/rbmfc8(28)500

Palavras-chave:

Obesidade

Nutrição em Saúde Pública

Hipertensão

Diabetes Mellitus

\section{Keywords:}

Obesity

Nutrition in Public Health

Hypertension

Diabetes Mellitus

\section{Palabras clave:}

Obesidad

Nutrición en Salud Pública

Hipertensión

Diabetes Mellitus

Fonte de financiamento:

PET-Saúde UFPA/Ananindeua

Parecer CEP: parecer no 164/11 do Instituto de Ciências da Saúde da Universidade Federal do Pará (ICS-UFPA) CAAE 0153.0.073.073-11 


\section{Introdução}

O consumo elevado de alimentos ricos em gordura saturada, colesterol e sódio, em conjunto com o sedentarismo, tem sido relacionado a uma gama de doenças crônicas/fatores de risco como hipertensão, dislipidemias e diabetes mellitus tipo 2 (DM-2), condiçóes que frequentemente estão associadas, na vida adulta ${ }^{1}$. A hipertensão arterial associa-se ao DM-2, à redução do HDL colesterol e ao aumento de triglicerídeos e, por isso, o tratamento clínico e os programas de prevenção não podem ignorar estas associaçóes, visto que os fatores de risco para as doenças crônicas não transmissíveis ocorrem de forma conjunta e interdependente ${ }^{2}$.

Apesar de consolidada a relação entre hipertensão arterial e fatores nutricionais, ainda náo estáo bem estabelecidos os mecanismos de atuação destes sobre a elevação da pressão arterial ${ }^{3}$, entretanto, são reconhecidos os efeitos que uma dieta saudável tem sobre o controle da pressão arterial. Entre os fatores nutricionais estudados associados à alta prevalência de hipertensão arterial, está o elevado consumo de álcool e sódio, bem como o excesso de massa corpórea ${ }^{4}$.

Inserido neste panorama, o diabetes mellitus representa também considerável encargo econômico para indivíduos e sociedade, especialmente quando mal controlado, sendo a maior parte dos custos diretos de seu tratamento relacionados a complicaçóes que comprometem a produtividade, a qualidade de vida e a sobrevida dos indivíduos. A progressiva ascensão das doenças crônicas no Brasil impóe a necessidade de uma revisão das políticas públicas em saúde, bem como a implantação de ações de saúde que incluam estratégias de redução de risco e controle dessas doenças5.

O adequado controle metabólico no DM-2 é fundamental para prevenir a ocorrência de episódios agudos de hiperglicemia ou hipoglicemia, como também para impedir ou retardar o desenvolvimento de complicaçóes como neuropatias, nefropatias, obesidade, dislipidemia e doenças cardiovasculares ${ }^{6}$.

Desta forma, o Ministério da Saúde em 1994, implantou o Programa Saúde da Família (PSF) com o objetivo de reorganizar a prática assistencial a partir da Atenção Primária à Saúde (APS). A dinâmica proposta, centrada na promoção da qualidade de vida e intervenção nos fatores de risco, permite melhor identificaçáo e acompanhamento dos indivíduos .

Considerando a importância do diagnóstico precoce e instituição de tratamento adequado para portadores de DM-2 e hipertensão e a fim de prevenir comorbidades, o conhecimento de fatores ambientais, biológicos, econômicos e sociais dessa população é imprescindível para determinação da melhor conduta terapêutica e fonte de estudos para atuação de políticas de saúde pública. O presente estudo procurou identificar a associação de condiçóes socioeconômicas, dados clínicos e hábitos alimentares sobre uma população hipertensa e/ou diabética.

\section{Métodos}

Estudo do tipo descritivo, quantitativo e transversal. A população amostral incluiu 125 indivíduos que faziam parte da população hipertensa e/ou diabética cadastrada e atendida pelas Estratégias Saúde da Família (ESFs) "Jardim Florestal” e "Mururé", no município de Ananindeua, Pará. Foram excluídos gestantes, menores de 18 anos, não cadastrados nessas ESFs e aqueles que não aceitaram participar da pesquisa.

A coleta dos dados ocorreu entre agosto de 2011 e fevereiro de 2012, sendo utilizado um questionário previamente elaborado contendo perguntas sobre o perfil socioeconômico, dados clínicos (peso, altura e índice de massa corpórea - IMC) e hábitos alimentares (frequência de consumo de frutas, verduras, legumes, carnes, etc.). Durante visitas domiciliares e em atividades educativas realizadas nas ESFs, os questionários foram aplicados pelos alunos e preceptores do Programa de Educação pelo Trabalho para a Saúde (PET-Saúde) da Universidade Federal do Pará. Todos os indivíduos foram orientados e esclarecidos previamente sobre o estudo e assinaram Termo de Consentimento Livre e Esclarecido (TCLE).

Para análise do perfil socioeconômico, foi utilizado o Critério de Classificação Econômica Brasil (CCEB) 2011 da Associação Brasileira de Empresas de Pesquisa $(\mathrm{ABEP})^{8}$. O CCEB confere pontos em função de cada característica domiciliar (presença e quantidade de alguns itens domiciliares de conforto e grau de escolaridade do chefe da família) e totaliza estes pontos. Em seguida, é feita uma correspondência entre faixas de pontuação do critério, distribuindo as pontuações em classificação econômica definidas por A1 - R\$ 14.366,00 (46 a 42 pontos); A2 - R \$ 8.099,00 (41 a 35 pontos); 
$\mathrm{B} 1$ - R\$ 4.558,00 (34 a 29 pontos); B2 - R \$ 2.327,00 (28 a 23 pontos); C1 - R\$ 1.391,00 (18 a 22 pontos); $\mathrm{C} 2$ - R\$ 933,00 (17 a 14 pontos); D - R\$ 618,00 (13 a 8 pontos); $\mathrm{E}-\mathrm{R} \$ 403,00$ (7 a 0 pontos).

Para a aferiçáo do peso, utilizou-se balança portátil calibrada, do tipo plataforma, marca Filizola ${ }^{\oplus}$, com capacidade de $150 \mathrm{~kg}$ e precisão de $100 \mathrm{~g}$, na qual o paciente deveria subir descalço e em posição ereta, braços estendidos ao lado do corpo. A unidade de medida utilizada foi o quilograma. Em relação à altura do indivíduo, utilizou-se fita antropométrica não flexível inelástica fixada a uma parede. Com o paciente em pé, descalço, com os calcanhares juntos, costas eretas, braços estendidos ao lado do corpo, olhando para a frente, sem fletir ou estender a cabeça, foi medida a altura encostando-se o paciente à parede na qual a fita estava afixada. Os valores foram representados em metros.

Para a classificação do estado nutricional por meio do IMC (razão entre o peso e o quadrado da altura), adotaram-se os valores de referência da Organização Mundial de Saúde (OMS) ${ }^{9}$ para adultos e idosos, que classifica: Magreza grau III (IMC $\leq 16,0)$; Magreza grau II (16,1 - 16,9); Magreza grau I (17,0 - 18,4); Eutrofia (18,5 - 24,9); Sobrepeso $(25,0$ - 29,9); Obesidade grau I $(30,0$ - 34,9); Obesidade grau II $(35,0$ - 39,9); Obesidade grau III ( $\geq 40)$. Quanto aos hábitos alimentares, utilizou-se o questionário do Guia Alimentar da População Brasileira ${ }^{10}$, elaborado pela Coordenação Geral de Alimentação e Nutrição (CGAN), com adaptaçóes.

Os resultados obtidos foram armazenados em banco de dados e, posteriormente, analisados, utilizando-se o Microsoft Office Excel 2007, o Word 2007 e o software Epi info, versão 3.5.1. O presente estudo seguiu as recomendaçôes do Conselho Nacional de Ética em Pesquisa (CONEP), sendo aprovado pelo Comitê de Ética e Pesquisa (CEP) do Instituto de Ciências da Saúde da Universidade Federal do Pará (ICS-UFPA), CAAE 0153.0.073.073-11 e parecer no 164/11.

\section{Resultados}

Os resultados demonstraram que, da população entrevistada: 66,4\% eram do sexo feminino, a média de idade foi 59,19 anos ( \pm 12 anos), sendo 65,6\% dos pacientes portadores de DM tipo 2 e 84,8\%, de hipertensão arterial.

De acordo com a Tabela 1, observa-se que a maioria (44,8\%) possuía ensino fundamental incompleto, tendo cursado regularmente entre 1 a 4 anos do ensino básico, seguido por 16\% que eram apenas alfabetizados, não tendo cursado escola regular, estando aptos apenas para leitura e escrita deficientes. Analisando os critérios de classificação econômica, observou-se, ainda, que $42,4 \%$ pertenciam à classe $\mathrm{C} 2$, com renda estimada em $\mathrm{R} \$ 933,00$; e $40 \%$ faziam parte da classe $\mathrm{D}$, com renda aproximada de $\mathrm{R} \$$ 618,00, de acordo com o CCEB 2011 da ABEP 8 .

Tabela 1. Perfil socioeconômico de pacientes atendidos em duas unidades de saúde no município de Ananindeua, Pará, 2011.

\begin{tabular}{lcc}
\hline \multicolumn{1}{c}{ Variáveis } & N & $\%$ \\
\hline Grau de escolaridade & & \\
Analfabeto & 12 & 9,6 \\
Alfabetizado & 20 & 16,0 \\
EFI & 56 & 44,8 \\
EFC & 11 & 8,8 \\
EMI & 5 & 4,0 \\
EMC & 17 & 13,6 \\
ESI & 3 & 2,4 \\
ESC & 1 & 0,8 \\
Classe econômica & & \\
Classe B2 & 5 & 4,0 \\
Classe C1 & 16 & 12,8 \\
Classe C2 & 53 & 42,4 \\
Classe D & 50 & 40,0 \\
Classe E & 1 & 0,8 \\
\hline
\end{tabular}

EFI: Ensino Fundamental Incompleto/ EFC: Ensino Fundamental Completo/ EMI: Ensino Médio Incompleto/ EMC: Ensino Médio Completo/ ESI: Ensino Superior Incompleto/ ESC: Ensino Superior Completo. B2: R\$ 2.327,00/ C1: R\$ 1.391,00/ C2: R\$ 933,00/ D: $R \$ 618,00 / E: R \$ 403,00$. 
A avaliação do estado nutricional mostrou IMC com média de 27,98 $( \pm 5,03) \mathrm{kg} / \mathrm{m}^{2}$. Quando classificados nutricionalmente (Tabela 2), observou-se que apenas $24,8 \%$ estavam com peso adequado e $1,6 \%$ foram classificados com Magreza grau I. A maioria $(73,6 \%)$ foi identificada com excesso de peso. O índice de obesidade atingiu $36 \%$ da população entrevistada, sendo que foi identificado um caso de obesidade grau III, também denominada obesidade mórbida, que corresponde a IMC $\geq 40 \mathrm{~kg} / \mathrm{m}^{2}$.

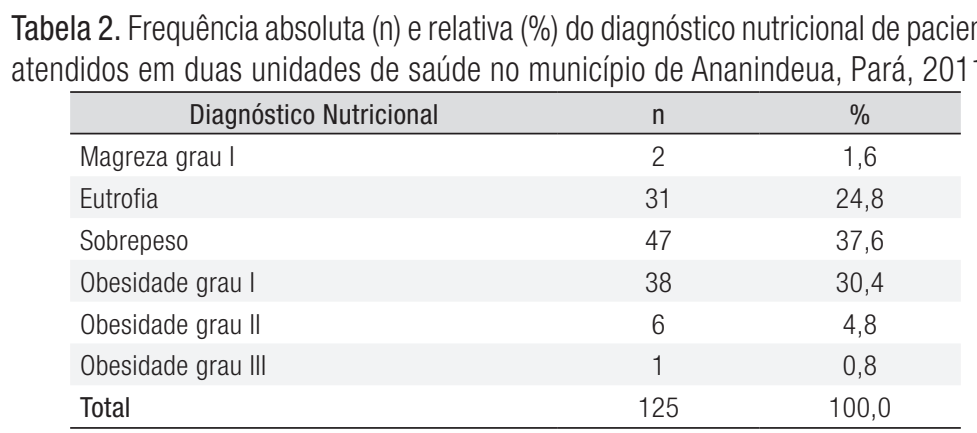

Quando relacionados o diagnóstico nutricional e classificação econômica dos entrevistados e diagnóstico nutricional em relação ao gênero, o p-valor não se mostrou relevante ( $\mathrm{p}$-valor $=0,177$ e 0,37 , respectivamente), indicando não haver diferença no estado nutricional entre as classes econômicas ou segundo o sexo. A pesquisa evidenciou ainda que a maioria $(81,6 \%, n=102)$ dos entrevistados alegou estar fazendo alguma dieta ou cuidado com a alimentaçáo para auxiliar no controle da pressão ou do diabetes mellitus.

Quando interrogados sobre os tipos de cuidados que estavam fazendo: 89,2\% afirmaram diminuir a quantidade de sal no preparo dos alimentos; $57,8 \%$ diminuíram o consumo de carnes vermelhas; $69,6 \%$ diminuíram o consumo de doces; $75,5 \%$ reduziram o consumo de frituras; e $41,2 \%$ diminuíram o consumo de alimentos com gordura. Convém ressaltar que os entrevistados poderiam atribuir mais de uma resposta à pergunta dirigida.

Analisando-se a frequência com que os participantes faziam as seis refeiçôes diárias, verificou-se que a maioria realizava as três principais refeiçóes: café da manhã (100\%); almoço (98,4\%); e jantar (71,2\%). Entretanto, quanto às outras refeições, observou-se baixa frequência, excetuando-se o lanche da tarde: 37,5\% faziam lanche da manhã; 70,4\%, lanche da tarde; e $31,5 \%$, o jantar. Observou-se que apenas 36\% da amostra do estudo faziam ao menos cinco refeiçóes diárias. Os resultados revelaram que 37,9\% dos entrevistados substituíam habitualmente as principais refeiçôes (almoço e jantar) por lanches e $12,9 \%$ afirmaram fazê-lo esporadicamente.

Em relação ao consumo de frutas, 32\% afirmaram consumir ao menos uma porção de frutas diariamente; já em relação ao consumo de saladas cruas e verduras e legumes cozidos, a maioria $(36,8 \%$ e $42,4 \%$, respectivamente) consumia apenas uma a duas vezes por semana. Vale acrescentar, ainda, que apenas $31,1 \%$ dos entrevistados consumiam feijáo diariamente e $28,7 \%$, uma a duas vezes durante a semana (Tabela 3 ).

Tabela 3. Frequência semanal de alguns alimentos consumidos pelos pacientes atendidos em duas unidades de saúde no município de Ananindeua, Pará, 2011.

\begin{tabular}{lcccccc}
\hline \multicolumn{1}{c}{ Tipo de alimento } & \multicolumn{5}{c}{ Frequência de consumo semanal $\mathrm{n}(\%)$} \\
\cline { 2 - 6 } & Todos 0s dias & 5 a 6 dias & 3 a 4 dias & 1 a 2 dias & Quase Nunca & Nunca \\
\hline Frutas & $40(32,0)$ & $8(6,4)$ & $24(19,2)$ & $33(26,4)$ & $18(14,4)$ & $2(1,6)$ \\
Saladas cruas & $16(12,8)$ & $4(3,2)$ & $19(15,2)$ & $46(36,8)$ & $28(22,4)$ & $12(9,6)$ \\
Verduras e legumes cozidos & $10(8,0)$ & $33(26,4)$ & $10(8,0)$ & $53(42,4)$ & $33(26,4)$ & $11(8,8)$ \\
Feijão & $38(31,1)$ & $8(6,6)$ & $21(17,2)$ & $35(28,7)$ & $11(9,0)$ & $9(7,4)$ \\
Peixes & $5(4,0)$ & $7(5,6)$ & $32(25,6)$ & $53(42,4)$ & $25(20,0)$ & $3(2,4)$ \\
Refrigerante & $11(8,8)$ & $2(1,6)$ & $11(8,8)$ & $29(23,2)$ & $38(30,4)$ & $34(27,2)$ \\
Frituras & $0(0,0)$ & $1(0,8)$ & $1(0,8)$ & $12(9,6)$ & $55(44,0)$ & $56(44,8)$ \\
Doces & $1(0,8)$ & $2(1,6)$ & $4(3,2)$ & $15(12,1)$ & $53(42,7)$ & $49(39,5)$ \\
\hline
\end{tabular}


O peixe era consumido uma a duas vezes por semana por 42,7\% dos entrevistados e $20 \%$ relataram quase nunca usá-lo na alimentação. Quanto à média do consumo de carne vermelha, 92,8\% confirmaram consumi-la regulamente, sendo que, destes, $68,9 \%$ retiravam o excesso de gordura das carnes. Já o consumo de frango foi de 99,2\%, sendo que, destes, 71,7\% retiravam a pele no momento do preparo (Tabela 4).

Quanto ao consumo de leite, apenas 27,2\% afirmaram consumi-lo frequentemente, enquanto $12 \%$ nunca o faziam e 60,8\% consumiam-no esporadicamente. Entre os que o consumiam, 75,4\% afirmaram consumi-lo na forma integral (Tabela 4).

Tabela 4. Hábitos alimentares de pacientes atendidos em duas unidades de saúde no município de Ananindeua, Pará, 2011.

\begin{tabular}{lcc}
\hline \multicolumn{1}{c}{ Variáveis } & $\mathrm{N}$ & $\%$ \\
\hline Carne vermelha & & \\
Sim & 116 & 92,8 \\
Não & 9 & 7,2 \\
Excesso de gordura da carne & & \\
$\quad$ Come carne com a gordura & 16 & 12,8 \\
$\quad$ Não come carne com muita gordura & 20 & 16,0 \\
$\quad$ Tira o excesso da gordura & 80 & 64,0 \\
Não se aplica & 9 & 7,2 \\
Frango & & \\
Sim & 124 & 99,2 \\
$\quad$ Não & 1 & 0,8 \\
Pele do frango & & \\
Come com a pele & 11 & 8,8 \\
Não come pedaços de frango com pele & 24 & 19,2 \\
Tira a pele do frango & 89 & 71,2 \\
$\quad$ Não se aplica & 1 & 0,8 \\
Leite & & \\
Sim & 34 & 27,2 \\
Às vezes & 76 & 60,8 \\
Não & 15 & 12,0 \\
Tipo de leite & & \\
Somente integral & 83 & 66,4 \\
Somente desnatado & 19 & 15,2 \\
Integral e desnatado & 8 & 6,4 \\
Não se aplica & 15 & 12,0 \\
\hline
\end{tabular}

Sobre o consumo semanal de refrigerantes (Tabela 3), 57,6\% afirmaram não possuir o hábito de consumi-los. Em relação às frituras, $88,8 \%$ dos entrevistados afirmaram não consumi-las. Além disso, quando indagados quanto ao tipo de gordura utilizada no preparo dos alimentos, 95,0\% relataram utilizar óleo vegetal. Quando analisada a frequência do consumo de doces ao longo da semana: $12,1 \%$ consumiam entre uma a duas vezes na semana, $42,7 \%$ quase nunca os consumiam e $39,5 \%$ nunca o faziam.

Em relação à adição de sal na comida já pronta, 91,2\% alegaram nunca fazê-lo. Quanto ao consumo de temperos industrializados e alimentos em conservas ao longo da semana, apenas 36\% nunca utilizavam temperos industrializados e 35,2\% utilizavam-nos diariamente nas suas preparaçóes. Em relação aos alimentos em conserva, 15,2\% os utilizavam pelo menos de uma a duas vezes na semana e $48,8 \%$, eventualmente.

Ao se relacionar gênero e dieta, observou-se diferença significativa ( $\mathrm{p}$-valor $<0,05)$, com predominância das mulheres entre os indivíduos que estavam fazendo alguma dieta ou cuidado com a alimentação, para auxiliar no controle da pressão ou do diabetes mellitus tipo 2 (Tabela 5). 
Tabela 5. Frequência absoluta (n) e relativa (\%) da associação entre as variáveis Dieta X Gênero de pacientes atendidos em duas unidades de saúde no município de Ananindeua, Pará, 2011.

\begin{tabular}{|c|c|c|c|c|}
\hline \multirow[t]{2}{*}{ Gênero } & Sem Dieta & Com Dieta & Total & \multirow[t]{2}{*}{$p$-valor } \\
\hline & \multicolumn{2}{|c|}{$n(\%)$} & & \\
\hline Feminino & $10(12,1)$ & $73(87,9)$ & $83(100,0)$ & \multirow{2}{*}{$0,018^{\#}$} \\
\hline Masculino & $13(31,0)$ & $29(69,0)$ & $42(100,0)$ & \\
\hline Totais & $23(18,4)$ & $102(81,6)$ & $125(100,0)$ & \\
\hline
\end{tabular}

\#Teste $\chi^{2}$ com correção de Yates/Demais análises cruzadas não se mostraram relevantes.

\section{Discussão}

Em 2005, a OMS estimava que $15 \%$ da população mundial era obesa ${ }^{11}$, enquanto, no Brasil, alguns dos resultados da seção de Antropometria e Estado Nutricional da Pesquisa de Orçamentos Familiares (POF) 2008-2009 revelaram que a prevalência de excesso de peso entre homens adultos era de 50,1\% e para mulheres era de $48 \%$, tendo a obesidade atingido $12,5 \%$ dos homens e $16,9 \%$ das mulheres ${ }^{12}$. No presente estudo, a prevalência do excesso de peso foi superior $(73,6 \%)$, o que é justificável, pois, uma vez que a população estudada era de risco (hipertensos e diabéticos), a obesidade tornou-se mais prevalente, dada sua relação com essas doenças crônicas. A falta de conhecimento sobre dieta balanceada e seus benefícios na redução dos fatores de risco para doenças crônicas não transmissíveis pode ter levado a população a atribuir a uma dieta balanceada somente a diminuição do consumo de sal, gordura e açúcar.

De acordo com os 10 Passos para Alimentação Saudável para Adultos propostos pelo MS, o ideal é que o indivíduo realize ao menos três refeições e dois lanches diariamente, entretanto apenas 36\% dos entrevistados realizavam cinco refeiçóes diárias $^{13}$. Tal artifício é ideal para manter o metabolismo basal acelerado e estimular a perda de peso. O maior fracionamento de refeiçôes constitui importante medida de controle/prevenção da dislipidemia e doenças cardiovasculares associadas. Ensaio clínico realizado com mulheres com excesso de peso (IMC $>25 \mathrm{~kg} / \mathrm{m} 2$ ), náo fumantes, com idades entre 30 e 50 anos e hipercolesterolêmicas (colesterol sérico > a $240 \mathrm{mg} / \mathrm{dL}$ ) demonstrou o efeito do aumento do número de refeições na redução do colesterol total sérico, em indivíduos que realizavam três ou mais refeiçóes diárias. Os dados mostraram-se relevantes, independentemente da idade, peso corporal e tipo de alimento ingerido ${ }^{14}$.

Um dado preocupante foi encontrado: $37,9 \%$ dos entrevistados afirmaram substituir habitualmente as principais refeiçôes (almoço e jantar) por lanches e 12,9\% o faziam esporadicamente. É necessário que as refeiçôes sejam completas, incluindo-se frutas, verduras e legumes, além de carboidratos, proteínas, gorduras, vitaminas e minerais, fornecidos adequadamente ${ }^{15}$.

Quando analisados os dados da pesquisa, verificou-se baixo consumo de frutas, verduras e legumes, no entanto a Food and Agriculture Organization (FAO) recomenda consumo diário de $400 \mathrm{~g}$ desses nutrientes, equivalendo, em média, a cinco porçóes. Quando consumidos diariamente e de forma regular, estes alimentos previnem o surgimento das principais doenças crônicas, tais como obesidade, doenças cardiovasculares e provavelmente, DM- $2^{16}$. Em populaçóes de baixa renda, impossibilitadas de comprar alguns alimentos, uma intervenção a ser considerada seria o incentivo ao cultivo de hortas domésticas ou comunitárias, contribuindo para a melhoria da alimentação desses indivíduos.

Apesar de o feijão ser um dos principais alimentos proteicos dos brasileiros, o resultado da pesquisa aponta baixo consumo pela populaçáo analisada. Trata-se de leguminosa muito importante, pois apresenta grande quantidade de fibras e minerais (especialmente ferro), além de possuir grande quantidade de proteínas. Um dos componentes do feijão que se destaca é a fibra solúvel betaglucana, auxiliar na prevenção de doenças cardiovasculares ${ }^{17}$.

O peixe é um alimento abundante na regiáo paraense, pois, além de saudável e nutritivo, quando comparado às demais carnes, contém baixo teor de gordura. Além disso, possui aminoácidos que ajudam a formar proteínas, ferro, vitamina B12, cálcio e lipídeos essenciais, importantes para o bom funcionamento do organismo ${ }^{18}$. Estudo de $1997^{19}$ verificou que o pescado constituía a principal fonte proteica de comunidades ribeirinhas em Monte Alegre, Pará. Entretanto, em outro estudo paraense de 1999, Isaac e Braga já alertavam para a necessidade de se melhorar rendimentos e minimizar perdas ecológicas e econômicas geradas pela pesca predatória ${ }^{20}$. A diminuiçáo da oferta ocasiona, por conseguinte, alta nos preços do pescado, especialmente em determinadas épocas do ano, dificultando a aquisição do peixe pela população metropolitana, o que pode ter refletido nos baixos percentuais de consumo obtidos na pesquisa.

Segundo este estudo, as carnes mais consumidas pela população eram a carne vermelha e aves. Entre os entrevistados, $92,8 \%$ relataram consumir carne vermelha, sendo que, destes, $68,9 \%$ retiravam o excesso de gordura, principal vilã para 
doenças cardiovasculares e não a carne em si, já que esta não traz malefícios para o coração quando consumida em quantidade adequada, sendo, inclusive, fonte de ferro-heme, de mais fácil absorção pelo organismo humano ${ }^{21}$.

O estudo mostrou ainda que 99,2\% dos entrevistados faziam consumo de carne de aves e, destes, 71,7\% retiravam a pele antes do preparo. Tal atitude é de fundamental importância, uma vez que a maior parte do colesterol presente no frango encontra-se na pele. Entretanto, quando comparada com a carne vermelha, o frango ainda é a melhor opção, pois possui proteínas de alto valor biológico e melhor digestibilidade ${ }^{22}$, além de ter menor custo na regiáo em que o estudo foi realizado.

O leite, apesar de não ser um alimento fundamental na vida adulta, é componente nutritivo para a dieta humana, devido à grande quantidade de cálcio e vitamina D. Em estudo que avaliou a densidade mineral óssea de mulheres brancas e idosas, observou-se que o consumo de leite, assim como a prática de exercícios físicos, eram fatores de proteçáo independentes para o desenvolvimento da osteoporose ${ }^{23}$. É um alimento útil também para manutenção da massa corpórea, com efeito benéfico na participação do metabolismo da glicose e no sistema renina-angiotensina, quando consumido na quantidade certa e com o teor de gordura reduzido ${ }^{24}$. Entretanto, o presente estudo revelou baixo consumo de leite diário (apenas 27,2\%), sendo que, destes, 75,4\% ainda o consumiam na forma integral, com altos teores de gordura. Esse quadro poderia ser revertido com o aumento do consumo em sua forma desnatada ou retirando-se a nata após fervura, diminuindo as taxas de gordura.

$\mathrm{Na}$ população pesquisada, apenas $27,2 \%$ afirmaram não consumir nenhum tipo de refrigerante. Já 30,4\% disseram quase nunca consumi-los e, destes, 65,6\% (quando o consumiam) faziam uso de refrigerantes não dietéticos. Recente estudo realizado em Pelotas-RS, com indivíduos adultos, observou que 20,4\% dos entrevistados consumiam refrigerantes não dietéticos regularmente, não havendo associação entre excesso de peso e consumo destes refrigerantes ${ }^{25}$. Entretanto, outros estudos evidenciam que bebidas açucaradas estão associadas ao aumento do peso. Uma hipótese seria o fato de os efeitos fisiológicos da saciedade serem diferentes para líquidos e para alimentos sólidos. Uma revisão de literatura, realizada com artigos publicados entre 1980 e 2008, concluiu que o estado físico do alimento pode influenciar o consumo alimentar e que alimentos líquidos exercem menor poder sacietógeno, em comparação aos sólidos. Atribui-se a isso: falta de mastigação, fase cefálica da ingestáo menos pronunciada, esvaziamento gástrico mais rápido e fatores cognitivos ${ }^{26}$. Dessa forma, o carboidrato ingerido em líquidos promoveria um balanço energético positivo maior, resultando em maior ganho de peso ${ }^{27}$.

O consumo de frituras observado na população estudada foi moderado, levando-se em conta os efeitos deletérios destes sobre o perfil lipídico e risco de aterosclerose. Entre os que consumiam, quando indagados sobre o tipo de gordura utilizada, 95\% afirmaram utilizar óleos vegetais, mais indicados para cocção de imersão, visto que não saturam facilmente; enquanto banhas, manteigas, margarinas e outros saturam rapidamente, não sendo adequados para longos períodos de frituras ${ }^{28}$. $\mathrm{O}$ consumo de alimentos ricos em açúcares simples também foi motivo de preocupação, tendo-se em vista os malefícios destes quando consumidos de forma excessiva, podendo aumentar a prevalência de excesso de peso ${ }^{29}$.

Para evitar o excesso do consumo de sal, 91,2\% dos entrevistados adotava a prática de não adicioná-lo após o preparo dos alimentos. Sabe-se que o consumo elevado de sal desencadeia vários problemas de saúde, principalmente a hipertensão arterial, que apresenta elevado risco de mortalidade pelas suas complicaçóes cardiovasculares ${ }^{30}$. Há de se levar em consideração que, apesar do baixo consumo de sal, grande parte dos entrevistados consumia temperos industrializados diariamente $(35,2 \%)$ ou eventualmente $(48,8 \%)$. Essas altas taxas refletem a falta de informação da população quanto à elevada presença de sódio nesses produtos, cuja finalidade vai desde acentuar o sabor até a conservação dos alimentos. No entanto, o seu uso indiscriminado vem aumentando as taxas de obesidade, esteatose hepática, diabetes mellitus, hipertensão arterial e dislipidemias ${ }^{30}$.

Observa-se também o incentivo da mídia pelo consumo de alimentos industrializados e de alto valor calórico. Estudos apontam nítida relação entre obesidade e o hábito de assistir televisão. Quanto mais horas se assiste à televisão, maior a probabilidade de se desenvolver obesidade, devido a três elementos envolvidos: a televisão ocupa as horas vagas, nas quais se poderia estar praticando atividade física; o indivíduo frequentemente come diante da televisão, não prestando atenção ao alimento e à quantidade ingerida; e grande parte da propaganda veiculada no horário nobre é dedicada à apresentaçáo de novas guloseimas. Esse fator apresenta-se relevante durante a infância, entretanto, convém salientar que uma criança obesa possui maiores chances de vir a se tornar um adulto também obeso e desenvolver hipertensão, diabetes e doenças cardiovasculares associadas ${ }^{31}$.

Estudo realizado no interior de São Paulo com amostra de população idosa apontou diferenças estatisticamente significantes na predominância de dieta desbalanceada na população de baixa renda, associada a escolaridade precária, sendo que as mulheres apresentavam ingestáo alimentar superior aos homens em lipídeos e energia ${ }^{32}$. Tais resultados não foram condizentes com o presente estudo, quando comparados diagnóstico nutricional e classificação econômica, mostrando não haver diferenças relevantes entre os dois grupos. A diferença entre os dois estudos pode ser devido à amostra selecionada, 
uma vez que, neste estudo, utilizaram-se dados de pacientes atendidos no Programa HIPERDIA de uma regiấo de baixa renda, não havendo diferença significativa entre indivíduos, ao contrário do estudo realizado em São Paulo, cuja amostra era de demanda espontânea de pacientes ambulatoriais. Outro estudo avaliou hipertensos e diabéticos usuários da ESF de Teixeiras-MG e evidenciou renda per capita mediana de 0,5 salários-mínimos, variando de 0,08 a 2,43 salários-mínimos. Entretanto, não foi correlacionada renda per capita com diagnóstico nutricional ${ }^{33}$.

Houve achados relevantes ao se relacionar gênero com realizaçáo de dieta. Segundo a literatura, no Brasil, a prevalência da obesidade é maior entre mulheres idosas do que em homens idosos, de acordo com a maioria dos levantamentos realizados com esta parcela da população ${ }^{32}$. Provavelmente, este fato está ligado às intensas modificaçôes hormonais femininas relacionadas à menopausa e ao eixo hipotalâmico-hipofisioadrenal, que regula a secreção de cortisol, e que pode afetar a composição corporal, levando ao acúmulo de gordura ${ }^{34}$. Pesquisa realizada com mulheres adultas pela Universidade Federal de Minas Gerais verificou que, entre estas, 16,3\% eram classificadas como sobrepeso e 26,5\% apresentavam algum grau de obesidade, sendo que $98,10 \%$ apresentavam percentual de gordura acima da média $(\geq 24 \%)^{35}$. Dados do MS informam ainda que a populaçáo adulta vem apresentando aumento na prevalência de excesso de peso em ambos os sexos ${ }^{36} \mathrm{e}$ os achados do presente estudo corroboram com essa tendência.

\section{Conclusão}

A maioria da populaçáo analisada apresentou prevalência de sobrepeso, ainda que afirmasse adotar dietas adequadas para controle da hipertensão arterial e diabetes mellitus. Esse resultado é, em parte, consequência da falta de conhecimento a respeito dos alimentos que devem ser restringidos e os que devem ser consumidos, pois equívocos foram encontrados nos hábitos alimentares diários da população, que desconhecia os malefícios da utilização de certos alimentos, tais como produtos industrializados, refrigerantes não dietéticos, baixo consumo de leite e a sua utilização na forma integral, baixo consumo de frutas e hortaliças, mau fracionamento das refeiçóes e substituição destas por lanches. Verifica-se a importância do desenvolvimento de estratégias de educação e promoção da saúde, visando incentivar mudanças nestes hábitos, além da necessidade de políticas públicas abrangentes para intervir no controle da qualidade dos alimentos ofertados.

Dessa forma, os resultados deste estudo induzem a necessidade de viabilizar meios para transferir o conhecimento produzido pelos pesquisadores/academia para os serviços de saúde (profissionais e usuários participantes do estudo) de modo a transformar a realidade desses indivíduos. Mediante essas informaçóes, torna-se necessário um acompanhamento mais intenso dos profissionais da área da saúde e a realização de programas educativos para a conscientização da comunidade quanto à importância da adoçáo de uma alimentação saudável e suas implicaçóes para o controle das doenças crônicas náo transmissíveis, beneficiando assim a qualidade de vida das pessoas.

\section{Referências}

1. Elias MC, Bolívar MSM, Fonseca FAH, Martinez TLR, Angelini J, Ferreira C, et al. Comparação do perfil lipídico, pressão arterial e aspectos nutricionais em adolescentes, filhos de hipertensos e de normotensos. Arq Bras Cardiol. 2004; 82(2): 139-42. http://dx.doi.org/10.1590/S0066-782X2004000200005

2. Sichieri R, Coitinho DC, Monteiro JB, Coutinho WF. Recomendações de alimentação e nutrição saudável para a população brasileira. Arq Bras Endocrinol Metab. 2000 Jun; 44(3): 227-32. http://dx.doi.org/10.1590/S0004-27302000000300007

3. Freitas OC, Carvalho FR, Neves JM, Veludo PK, Parreira RS, Gonçalves RM, et al. Prevalence of Hypertension in the urban population of Catanduva, in the state of São Paulo, Brazil. Arq Bras Cardiol. 2001 Jul; 77: 16-21. http://dx.doi.org/10.1590/S0066-782X2001000700002

4. Molina MCB, Cunha RS, Herkenhoff LF, Mill JG. Hipertensão arterial e consumo de sal em população urbana. Rev Saúde Pública. 2003; 37(6): 743-50.

5. Portero KCC, Motta DG, Campino AAC. Abordagem econômica e fluxograma do atendimento a pessoas com Diabetes Mellitus tipo 2 na rede pública de saúde de um município paulista. Saúde Rev. 2003; 5(11): 35-42.

6. Burnet DL, Cooper AJ, Drum ML, Lipton RB. Risk factor for mortality in a diverse cohort of patients with childhood-onset diabetes in Chicago. Diabetes Care. 2007; 30(10): 2559-63. http://dx.doi.org/10.2337/dc07-0216

7. Brasil. Departamento de Atenção Básica, Secretaria de Políticas de Saúde. Programa Saúde da Família. Rev Saúde Pública. $2000 ; 34: 316$.

8. Associação Brasileira de Empresas de Pesquisa - ABEP. Critério de Classificação Econômica Brasil. São Paulo; 2011 [online]. [acesso em 2011 Jun 15]. Disponível em: http://www.abep.org/novo/Content.aspx?ContentID=302 
9. World Health Organization - WHO. Diet, nutrition, and the prevention of chronic diseases. Geneva: World Health Organization; 1990. (WHO Technical Report Series 797).

10. Brasil. Ministério da Saúde. Guia Alimentar da População Brasileira. Coordenação Geral de Alimentação e Nutrição - CGAN. [acesso em 2011 Jun 15]. Disponível em: http://189.28.128.100/nutricao/docs/geral/guia_alimentar_bolso.pdf

11. Organização Mundial da Saúde - OMS. Prevenção de doenças crônicas: um investimento vital. Brasília: Organização Mundial da Saúde; 2005.

12. Instituto Brasileiro Geografia E Estatística - IBGE. POF 2008-2009: desnutrição cai e peso das crianças brasileiras ultrapassa padrão internacional. 27 Ago 2010. [acesso em 2012 Mar 14]. Disponível em: http://www.ibge.gov.br

13. Brasil. Ministério da Saúde. 10 passos para a alimentação saudável. [acesso em 2012 Mar 16]. Disponível em: 189.28.128.100/nutricao/docs/ geral/10passosAdultos.pdf

14. Oliveira MC, Sichieri R. Fracionamento das refeições e colesterol sérico em mulheres com dieta adicionada de frutas ou fibras. Rev Nutr. 2004; 17(4): 449-59. http://dx.doi.org/10.1590/S1415-52732004000400005

15. Stella RH. Jantar balanceado deixa seu metabolismo mais rápido. 2009 [online] [acesso em 2012 Mar 16] Disponível em: http://www.minhavida.com.br

16. Organização das Nações Unidas para Agricultura e Alimentação. [acesso em 2012 Mar 09] Disponível em: https://www.fao.org.br/

17. Vilarta R, Sonati JG, organizadores. Diagnóstico da Alimentação Saudável e Atividade Física na Fundação de Desenvolvimento da Unicamp. Campinas: IPES Editorial; 2007. 95 p.

18. Brasil. Ministério da Saúde. Ministério da Pesca e Agricultura. Inclua pescado na sua alimentação. É gostoso e faz bem para a saúde. [acesso 2012 Mar 09] Disponível em: http://portal.saude.gov.br/saude/campanha/semana_peixe_cartilha_090911.pdf

19. Cerdeira RGP, Ruffino ML, Isaac VJ. Consumo de Pescado e Outros Alimentos pela População Ribeirinha do Lago Grande de Monte Alegre, PA-Brasil. Acta Amazonica 1997; 27(3): 213-228.

20. Isaac VJ, Braga TMP. Rejeição de Pescado nas Pescarias da Região Norte do Brasil. Arq. Ciên. Mar 1999; 32: 39-54.

21. Silva RS. Carne vermelha não é vilã da dieta. 2007. [acesso 2012 Mar 09] Disponível em: http://cyberdiet.terra.com.br/carne-vermelha-nao-e-vilada-dieta-2-1-1-530.html

22. Carne Vermelha versus Carne Branca Saúde \& Qualidade de Vida - Saúde \& Nutrição. RgNutri. [acesso em 2012 Mar 9] Disponível em: http://www. rgnutri.com.br/sqv/saude/cvcb.php

23. Frazão P, Naveira M. Fatores associados à baixa densidade mineral óssea em mulheres brancas. Rev Saúde Pública. 2007; 41(5): 740-48. http:// dx.doi.org/10.1590/S0034-89102007000500008

24. Martini LA, Wood RJ. Ingestão de leite e risco de diabetes melito tipo 2, hipertensão e câncer de próstata. ArqBrasEndocrinolMetab. 2009 Jul; 53(5): 688-694. http://dx.doi.org/10.1590/S0004-27302009000500021

25. Rombaldi AJ, Neutzling MB, Silva MC, Azevedo MR, Hallal PC. Fatores associados ao consumo regular de refrigerante não dietético em adultos de Pelotas, RS. Rev Saúde Pública. 2011; 45(2): 382-90. http://dx.doi.org/10.1590/S0034-89102011005000009

26. Mourão DM, Bressan J. Influência de Alimentos Líquidos e Sólidos no Controle do Apetite. Rev Nutr. 2009; 22(4): 537-547. http://dx.doi.org/10.1590/ S1415-52732009000400009

27. DiMeglio DP, Mattes RD. Liquid versus solid carbohydrate: effects on food intake and body weight. Int J Obes Relat Metab Disord. 2000; 24(6): 794-800. http://dx.doi.org/10.1038/sj.ijo.0801229

28. Philippi, Sônia Tucunduva. Nutrição e técnica dietética. Barueri: Manole; 2006. 402 p.

29. Carmo MB, Toral N, Silva MV, Slater B. Consumo de doces, refrigerantes e bebidas com adição de açúcar entre adolescentes da rede pública de ensino de Piracicaba, São Paulo. Rev Bras Epidemiol. 2006 Mar; 9(1): 121-130. http://dx.doi.org/10.1590/S1415-790X2006000100015

30. Piati J, Felicetti CR, Lopes AC. Perfil Nutricional de Hipertensos Acompanhados pelo Hiperdia em Unidade Básica de Saúde de Cidade Paranaense. Rev Bras Hipertens. 2009; 16(2): 123-129.

31. Damiani D, Carvalho DP, Oliveira RG. Obesidade na infância - um grande desafio! Pediatr Mod. 2000; 36(8).

32. Cabrera MAS, Jacob W F'. Obesidade em idosos: prevalência, distribuição e associação com hábitos e co-morbidades. Arq Bras Endocrinol Metab. 2001; 45(5).

33. Cotta RMM, Reis RS, Batista KCS, Dias G, Alfenas RCG, Castro FAF. Hábitos e práticas alimentares de hipertensos e diabéticos: repensando o cuidado a partir da atenção primária. Rev Nutr. 2009; 22(6): 823-835.

34. Kac G, Velásquez-Meléndez G, Coelho MASC. Fatores associados à obesidade abdominal em mulheres em idade reprodutiva. Rev Saúde Pública. 2001; 35(1): 46-51. http://dx.doi.org/10.1590/S0034-89102001000100007

35. Esteves EA, Ávila MVP, Almeida FZ. Ingestão calórica e relações entre ingestão e variáveis de adiposidade em mulheres adultas. Alim Nutr Araraquara. 2010 Out-Dez; 21(4)

36. Instituto Brasileiro de Geografia e Estatística - IBGE. Pesquisa de orçamentos familiares 2008-2009: antropometria e estado nutricional de crianças, adolescentes e adultos no Brasil: análise dos resultados. [acesso 2012 Mar 10] Disponível em: http://www.ibge.gov.br 